\title{
Childhood asthma and physical activity: a systematic review with meta-analysis and Graphic Appraisal Tool for Epidemiology assessment
}

\author{
Lene Lochte $^{1 *}$ (D) Kim G. Nielsen ${ }^{2}$, Poul Erik Petersen ${ }^{1}$ and Thomas A. E. Platts-Mills ${ }^{3}$
}

\begin{abstract}
Background: Childhood asthma is a global problem affecting the respiratory health of children. Physical activity (PA) plays a role in the relationship between asthma and respiratory health. We hypothesized that a low level of PA would be associated with asthma in children and adolescents. The objectives of our study were to (1) summarize the evidence available on associations between PA and asthma prevalence in children and adolescents and (2) assess the role of PA in new-onset or incident asthma among children and adolescents.
\end{abstract}

Methods: We searched Medline, the Cochrane Library, and Embase and extracted data from original articles that met the inclusion criteria. Summary odds ratios (ORs) and confidence intervals (Cls) were used to express the results of the meta-analysis (forest plot). We explored heterogeneity using funnel plots and the Graphic Appraisal Tool for Epidemiology (GATE).

Results: We retrieved 1,571 titles and selected 11 articles describing three cohort and eight cross-sectional studies for inclusion. A meta-analysis of the cohort studies revealed a risk of new-onset asthma in children with low PA (OR [95 \% Cl] 1.32 [0.95; 1.84] [random effects] and 1.35 [1.13; 1.62] [fixed effects]). Three cross-sectional studies identified significant positive associations between childhood asthma or asthma symptoms and low PA.

Conclusions: Children and adolescents with low PA levels had an increased risk of new-onset asthma, and some had a higher risk of current asthma/or wheezing; however, there was some heterogeneity among the studies. This review reveals a critical need for future longitudinal assessments of low PA, its mechanisms, and its implications for incident asthma in children. The systematic review was prospectively registered at PROSPERO (registration number: CRD42014013761; available at: http://www.crd.york.ac.uk/PROSPERO [accessed: 24 March 2016]).

Keywords: Systematic review, Pediatric, Asthmatic disease, Exercise

\section{Background}

Asthma is one of the most common chronic pediatric diseases [1]. The prevalence of asthma in children has increased over the last thirty years in most developed countries [2,3], although the prevalence has started to decrease in adolescents in Western countries [4, 5]. The etiology of childhood asthma is still not understood $[6,7]$, and the increase in prevalence has not been fully

\footnotetext{
* Correspondence: rkb664@alumni.ku.dk

'Department of Odontology, University of Copenhagen, Copenhagen 1014, Denmark

Full list of author information is available at the end of the article
}

explained [8]. Physical activity (PA) is known to be associated with asthma symptoms in asthmatic children $[9,10]$, but its role in asthma prevention is unclear.

In Europe, PA levels have declined in children and adolescents [11]. Physical conditioning programs may reduce childhood asthma symptoms [12-14]; moreover, studies of asthmatic children have indicated that PA may induce anti-inflammatory effects $[15,16]$ such that brief intervals of PA alter the immune response [15]. However, whether such effects $[17,18]$ translate into a reduced risk of developing asthma also remains unclear. 
The decline in PA may be linked to the increased prevalence and severity of childhood asthma [7, 9, 19, 20] or even to undiagnosed asthma [21]. Cross-sectional studies have shown inconsistent associations between PA and childhood asthma. In some studies, low levels of PA were related to a high asthma risk [22-24]; however, other studies did not find an association [25]. The few longitudinal studies on PA and childhood asthma have produced diverse results; in fact, one study showed that high levels of PA were related to an increase in diagnosed asthma [26].

Few authors [27] have collated the results of observational studies in this field. Therefore, our objectives were to (1) summarize the available evidence on associations between PA and asthma prevalence in children and adolescents and (2) assess the role of PA in new-onset or incident asthma in children and adolescents. We report the hypothesized associations between low PA and asthma in children and adolescents.

\section{Methods \\ Design}

This study was a systematic literature review that included a quantitative analysis (meta-analysis) and assessments using the Graphic Appraisal Tool for Epidemiology (GATE) [28]. We identified published studies examining the associations between PA and asthma in children and adolescents.

The protocol followed the Centre for Reviews and Dissemination (CRD) guidelines [29] for conducting systematic reviews: we (1) identified the available research and selected studies for inclusion, (2) extracted data, (3) assessed and described study quality, and (4) synthesized our findings. The reporting of our findings adhered to the Preferred Reporting Items for Systematic reviews and Meta-Analyses (PRISMA) statement [30] and, initially, to the consensus statement of the Meta-analysis Of Observational Studies in Epidemiology (MOOSE) Group [31]. Additional file 1 presents the PRISMA [32] checklist items that we examined. Additional file 2 presents the details obtained from using the Reporting Checklist of the MOOSE Group [31]. We used the GATE approach [28] to illustrate and assess the quality of the studies that did not qualify for the meta-analysis. When possible, we summarized the individual quality of these studies, assessing errors, effect sizes, and study applicability. For the meta-analysis, we used data on exposure to PA provided for asthma and control children; the outcomes were new-onset childhood asthma/or wheezing.

\section{Ethical aspects}

Since this is a systematic review based on published literature, the ethical requirements have been met previously for each individual study. Accordingly, the relevant approvals are stated in each original publication (article) included in our review. Written informed consent was obtained from the patient's guardian/parent/next of kin for the publication of each original article included in this report and any accompanying images.

Inclusion criteria for studies on PA and asthma diagnoses We included longitudinal and cross-sectional studies that investigated asthma and PA in children and adolescents aged $0-18$ years. PA was documented by either interviews or self-administered questionnaires. Childhood asthma was defined using parental reports of either physician diagnosis of asthma, "current" (within last 12 months) asthma, "ever" (lifetime) asthma, wheezing, exercise-induced asthma (EIA), or medical treatment of asthma symptoms. We defined new-onset asthma (incident asthma) as a physician diagnosis of asthma/or wheezing. Hence, for incident asthma, there was no sampling based on disease status [33]. We used asthma/or wheezing (a representative asthma symptom) [34] to capture the heterogeneous symptomatology of asthma in children [35].

We defined PA as a behavioral concept that varied according to "leisure time" or "sports and exercise" [36]. We recognized that PA can be further characterized by its dimensions as follows: (1) frequency, (2) intensity, (3) duration, and (4) type [37]. Intensity has been identified as the key dimension for possible dose-response relationships with either reduced or increased health risks for exerciseinduced medical conditions [38]. This review did not distinguish between PA and exercise. The concept "PA" referred to general leisure-time $\mathrm{PA}$, exercise, or sports during or outside of school hours [39]. High amounts of TV viewing (duration in hours) represented sedentary behavior [40, 41] and were used as a proxy for low PA. This approach was based on the previous use of TV viewing $[24,42]$ which validated that TV viewing could be used to represent PA in population surveys. It was beyond the scope of this review to discuss the scientific distinctions between sedentary activity and physical inactivity in children and adolescents.

\section{Inclusion criteria for the meta-analysis}

We adhered to appropriate standards [29] in defining our criteria for the meta-analysis, which were as follows: (1) broadly similar research questions, (2) comparable participant populations (children and adolescents), and (3) broadly similar research mechanisms.

\section{Exclusion criteria}

We excluded studies involving adults $>18$ years of age and non-English-language studies [43]. We also excluded single outcomes of intermediate phenotypes for childhood asthma (i.e., bronchial hyperresponsiveness [BHR], allergic rhinoconjunctivitis, atopic dermatitis, airway inflammation, eczema) and cumulative incidence along with studies that had fitness or body composition as 
their only outcomes. Studies that reported on only PA or asthma were excluded, as were clinical investigations (e.g., randomized controlled trial [RCT] designs) of training and/or medical treatment in children with asthma. If pediatric asthma or PA was explored using noncomparable (rare) methodologies or the studies excluded relevant participants, the studies were excluded. We excluded other reviews, methodology reports, validation studies, and studies that collected data for other purposes or had other non-applicable outcomes. The two stages of exclusion are illustrated in Fig. 1, and the articles excluded at each stage are grouped by exclusion rationale in Additional file $3 \mathrm{~A}$ and $\mathrm{B}$.

\section{Search strategy}

\section{Identifying studies and study selection}

We searched the following databases: Medline, National Library of Medicine (1946 to the last search date: 7 Jan 2014), the Cochrane Library (all Cochrane products to the last search date: 13 Jan 2014), and Embase/Excerpta Medica (2013 to the last search date: 17 Jan 2014). We used medical subject headings (MeSH) for asthma/or wheezing and PA. In Medline, "physical activity" was not available as a $\mathrm{MeSH}$ heading, and therefore we included the MeSH headings "physical fitness", "exercise", and "physical exertion"; we also restricted the search to English language, humans, and age $0-18$ years. Table 1 illustrates the full electronic search strategy used in

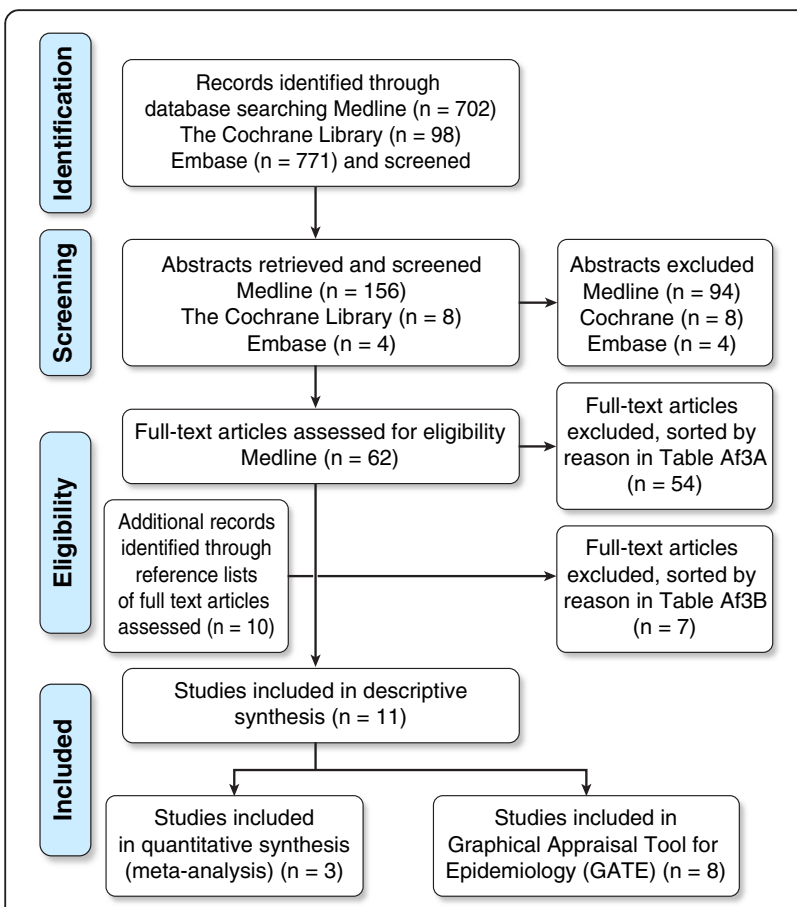

Fig. 1 Inclusion and Exclusion Criteria for Systematic Reviews. Numbers of search results from Medline, the Cochrane Library, and Embase
Table 1 Full Electronic Search Strategy for Medline

\begin{tabular}{ll}
\hline Action & Term \\
\hline 1 & asthma \\
3 & abronchial hyperreactivity \\
4 & abronchoconstriction \\
5 & ${ }^{a}$ respiratory hypersensitivity \\
6 & arespiratory sounds \\
7 & adyspnea \\
8 & asthma, exercise-induced \\
9 & arespiratory function tests \\
10 & aexercise test \\
11 & 1 or 2 or 3 or 4 or 5 or 6 or 7 or 8 or 9 \\
12 & aphysical fitness \\
13 & aexercise \\
14 & aphysical exertion \\
15 & 11 or 12 or 13 \\
16 & 10 and 14 \\
17 & Limit 15 to English language \\
18 & Limit 16 to humans \\
\hline & Limit 17 to "all child (0 to 18 years)"
\end{tabular}

andicates a focused search using medical subject heading (MeSH) terms. "Or" was used to combine related search terms. "And" was used to combine two sets of terms for asthma and physical activity

Medline. Initially, to expand the search, we conducted exploratory text, title, and adjacent word searches. Because we obtained large numbers of unrelated titles, these searches were subsequently omitted. One medical subject librarian (CFB) reviewed our search strategies for the Cochrane and Medline databases to ensure that the variation in search terms across the databases was taken into account. We read review articles and identified additional studies from the reference lists of retrieved full-text articles.

LL searched and screened studies by title and abstract for eligibility. Two medical students declined to be independent reviewers, and LL identified the articles for inclusion. When necessary, assessment was performed by the lead investigator (PEP). Figure 1 presents a flow diagram illustrating the studies identified by the database searches.

\section{Data extraction and study quality}

LL extracted information from the included studies. Table 2 shows the information points that were extracted from each study for the descriptive data synthesis. The extracted items represented adopted standards for methods, participants, outcomes, and results as defined in the checklist of The Cochrane Handbook for Systematic Review [44]. For the quantitative data synthesis (the meta-analysis), we extracted individual summary data [29] from each study that 
Table 2 Data Extracted from Individual Studies in the Systematic Review

\begin{tabular}{l}
\hline Data \\
\hline Name of first author \\
Year of publication \\
Study design \\
Age (years) of study population: Mean ( $\pm 2 \mathrm{SD}$ ) or range \\
Definition of physical activity \\
Definition of asthma \\
Number of children with asthma and total study population size \\
Main effect size and confidence interval \\
Adjustment covariates \\
Key conclusions of the study authors
\end{tabular}

met the criteria for meta-analysis. We excluded BHR as an asthma phenotype and consequently were only able to obtain asthma severity data from a few of the reviewed studies [22, 24, 45].

Using GATE [28] entailed documenting the study population, representativeness, measurement(s), and timing. All data that were extracted to electronic GATE forms [46] are illustrated in Additional file 4.

\section{Statistical methods}

The studies we examined followed different protocols, and therefore, we explored the clinical and methodological sources of their heterogeneity by reviewing the descriptive study characteristics that we extracted (Table 2). For the meta-analysis, we reported both random- and fixed-effects models (using inverse variance [29]) to illustrate the respective inter- and intra-study variability [47]. Technically, we produced $2 \times 2$ tables; i.e., we entered the numbers of children who developed asthma in the exposed (low PA) and unexposed (high PA) groups [48]. This approach produced summary statistics for each individual study and an overall estimate, both of which were expressed as odds ratios (ORs) and $95 \%$ confidence intervals (CIs). Forest plots were used to illustrate these summary statistics and the variation (heterogeneity) across the studies. We expressed the percentages of variability in the effect estimates that were attributable to between-study variation (heterogeneity) rather than chance using I-squared $\left(\mathrm{I}^{2}\right)$, and the statistical assessment was performed using the chi-squared $\left(x^{2}\right)$ test $[29,47]$. We assessed the risk of publication bias or selective outcome reporting [30] across studies by estimating the standard errors (SEs) of the logarithmic $(\log )$ scale ORs $(\log \mathrm{ORs})$, and we depicted these graphically on the horizontal (logORs) and vertical (SEs) axes of a funnel plot. In addition, we assessed the funnel plot for asymmetry [49]. We used STATA ${ }^{\mathrm{ma}}$ version 12 (StataCorp, College Station, TX, US) [50] for the calculations and $P$ set at $5 \%$.

\section{Results}

\section{Identified studies}

The searches yielded a total of 1,571 titles, and 11 studies that examined PA and childhood asthma met the inclusion criteria. Initially, we removed duplicates and contacted the authors of two articles to clarify details regarding the original data. Both authors responded, and we obtained the full texts of 62 studies. Of the 11 studies that met the inclusion criteria, three were cohort studies $[45,51,52]$, and eight were cross-sectional studies $[9,22-25,53-55]$. We excluded 54 studies followed by seven additional studies at two different stages (Fig. 1). Tables 3 and 4 present the data extracted from each study sorted by study design. Below follow reports on the cohort studies (including meta-analysis) and the cross-sectional studies given in separate sections.

\section{Cohort studies}

Measurements of new-onset (incident) asthma/or wheezing Two studies [45, 51] described cases of new-onset asthma using a physician's diagnosis of asthma (Table 3), and one study described new-onset wheezing [52]. We synthesized three cohort studies $[45,51,52]$ that met the criteria for inclusion in our meta-analysis. The follow-up times (in years) were 6-7 [52], 10 [51], and 11.5 [45] (Table 3). In these studies [45, 51, 52], a total of 549 children had new-onset asthma/or wheezing, and the total number of cohort children studied was 6,037 (Table 3). The reported asthma prevalence was $6.0 \%$ [45], the new-onset wheezing prevalence was $11.3 \%$ [52], and the asthma incidence rate was $16.6 \%$ per 1,000 person-years [51]. Overall, $57.7 \%$ (317) of the cohort children with new-onset asthma/or wheezing had low PA $[45,51,52]$ (Table 5).

\section{Results from meta-analysis}

We conducted a meta-analysis using data on asthma and PA provided by three articles $[45,51,52]$. To combine the study results, we reclassified the exposure variables. The original PA variables were number of team sports played (none, 1-2, $>2$ ) [51], sports participation frequency ( $\leq$ once per month, sonce per week, $2-3$ times per week, $>3$ times per week) [52], and duration of TV viewing (not at all, $<1$ hour per day, 1-2 hours per day, $>2$ hours per day) [45]; for the meta-analysis, we dichotomized the results into no team sports played (low PA) and $\geq 1$ team sport played (high PA) [51], sports participation $\leq$ once per week (low PA) and $\geq 2$ times per week (high PA) [52], and TV viewing $\geq 1$ hour per day (low PA) and $<1$ hour per day (high PA) [45]. The reference category was high PA in both the random- and fixed-effects models. The overall meta-analysis results showed positive risks for newonset asthma (OR [95 \% CI] 1.32 [0.95; 1.84] [random effects] and 1.35 [1.13; 1.62] [fixed effects]) in children with low PA compared with high PA (reference). These 
Table 3 Cohort or Longitudinal Studies Included in the Systematic Review by Selected Study Information

\begin{tabular}{|c|c|c|c|c|c|c|c|c|c|c|}
\hline \multirow[t]{2}{*}{ Author } & \multirow[t]{2}{*}{ Year } & \multirow{2}{*}{$\begin{array}{l}\text { Study } \\
\text { design }\end{array}$} & \multirow{2}{*}{$\begin{array}{l}\text { Age }^{a} \\
\text { (years) }\end{array}$} & \multicolumn{2}{|l|}{ Definition } & \multicolumn{2}{|l|}{ Number } & \multirow{2}{*}{$\begin{array}{l}\text { Main effect size Adj } \\
\text { OR/HR/GMR/mean }\end{array}$} & \multirow[t]{2}{*}{ Adj covariates } & \multirow{2}{*}{$\begin{array}{l}\text { Key conclusions } \\
\text { reported by the } \\
\text { study authors }\end{array}$} \\
\hline & & & & $\begin{array}{l}\text { Physical } \\
\text { activity }\end{array}$ & Asthma & Asthma & $\overline{\text { Total }}$ & & & \\
\hline Vogelberg & 2007 & $\begin{array}{l}\text { Cohort } \\
\text { follow-up } \\
6-7 \text { years }\end{array}$ & $16-18$ & $\begin{array}{l}\text { Sports freq } \\
\text { (questionnaire) }\end{array}$ & $\begin{array}{l}\text { Physician } \\
\text { diagnosed (wz) }\end{array}$ & $329^{w z, b}$ & $2,858^{b}$ & $\begin{array}{l}\text { Risk (OR [95 \% Cl]) } \\
\text { of incident wz by } \\
\text { sports > } 3 \text { times per } \\
\text { wk vs } \leq \text { once per } \\
\text { month (rfgr): } 0.8 \\
(0.5-1.3)\end{array}$ & $\begin{array}{l}\text { Active and passive } \\
\text { smoking, BMI, } \\
\text { SES, gender }\end{array}$ & $\begin{array}{l}\text { Inverse associations } \\
\text { between WZ and } \\
\text { sport or PC }\end{array}$ \\
\hline Sherriff & 2009 & $\begin{array}{l}\text { Cohort } \\
\text { follow-up } \\
11.5 \text { years }\end{array}$ & 11.5 & $\begin{array}{l}\text { TV viewing } \\
\text { (questionnaire) }\end{array}$ & $\begin{array}{l}\text { Physician } \\
\text { diagnosed }\end{array}$ & $78^{b}$ & $1,599^{b}$ & $\begin{array}{l}\text { Associations (OR } \\
[95 \% \mathrm{Cl}]) \text { of asthma } \\
\text { at age } 11.5 \text { years with } \\
\text { TV viewing at age } 3.5 \\
\text { years ( }>2 \text { hrs/day) vs } \\
1-2 \text { hrs/day (rfgr): } 1.8 \\
(1.2-2.6) \\
(P \text { trend }=0.0003)\end{array}$ & $\begin{array}{l}\text { BMI, maternal asthma/ } \\
\text { allergies and smoking, } \\
\text { social variables }\end{array}$ & $\begin{array}{l}\text { Longer duration of TV } \\
\text { viewing associated } \\
\text { with development of } \\
\text { asthma in later } \\
\text { childhood }\end{array}$ \\
\hline Islam & 2009 & $\begin{array}{l}\text { Cohort } \\
\text { follow-up } \\
10 \text { years }\end{array}$ & $7-11+$ & $\begin{array}{l}\text { Team sports } \\
\text { (questionnaire) }\end{array}$ & $\begin{array}{l}\text { Physician } \\
\text { diagnosed }\end{array}$ & $142^{b}$ & $1,580^{b}$ & $\begin{array}{l}\text { Associations (HR } \\
\text { [95\% } \% \text { Cl]) of GSTP1 }{ }^{c} \\
\text { genotypes with new- } \\
\text { onset asthma by }>\text { two } \\
\text { team sports vs none } \\
\text { (rfgr): } 2.66(1.2-5.9) \\
(P<0.05)^{C} \text { Subclass } \\
\text { of GST }\end{array}$ & $\begin{array}{l}\text { Ethnicity, community } \\
\text { of residence, genetic } \\
\text { information (GSTM1 } \\
\text { and SNP1/SNP3) } \\
\text { 'Subclass of GST }\end{array}$ & $\begin{array}{l}\text { Children with Val }{ }^{105} \\
\text { variant allele may be } \\
\text { protected against } \\
\text { increased risk of } \\
\text { asthma by exercise }\end{array}$ \\
\hline
\end{tabular}

Adj Adjusted or adjustment, BMI Body mass index, Cl Confidence interval, Freq Frequency, GST Glutathione S-transferase, Hr/hrs Hour/hours, HR Hazard ratio, OR

Odds ratio, Rfgr Reference group, SD Standard deviation, SES Socio-economic status, SNP Single nucleotide polymorphism, Vs Versus, Wk Week, Wz Wheezing

${ }^{a}$ Age: Mean $( \pm 2 \mathrm{SD})$ or range

${ }^{\mathrm{b}}$ Those who contributed data on asthma/wheezing and physical activity to the meta-analysis

'Subclass of GST

results are illustrated in Fig. 2 (random effects) and Fig. 3 (fixed effects). $\mathrm{I}^{2}$ was $60.6 \%\left(\mathrm{X}^{2}=5.08, P=0.079\right)$ for both random and fixed effects.

\section{Consistency of meta-analysis results: risk of bias across studies}

In Fig. 4, the studies that included larger numbers of asthmatic participants $[51,52]$ were positioned toward the top, i.e., the upper two-thirds of the funnel, representing large sample sizes and small standard errors. Figure 4 also shows that the studies in the meta-analysis $[45,51,52]$ were within the $95 \%$ confidence limits (diagonal, dashed lines) around the summary estimate.

\section{Validity and quality: risk of bias within studies}

Our review showed that these three studies [45, 51, 52] explored the role of the temporal sequence following quantified PA exposure and its effect on new-onset asthma/or wheezing in children and adolescents.

\section{Cross-sectional studies}

\section{Measurements of current or ever (prevalent) asthma/or} wheezing

As shown in Table 4, two studies [22, 53] defined current asthma using questionnaires and a medical provider or physician diagnosis of asthma, whereas a majority $[9,23-25,54,55]$ used the International Study of Asthma and Allergies in Childhood (ISAAC) definitions.
Validity and quality: risk of bias within studies

We applied the GATE approach developed for the critical appraisal of quantitative studies (electronic forms) $[28,46]$. When data were available, we first extracted study numbers regarding exposure, comparison, and outcomes for the association between PA and childhood asthma (Additional file 4). We first used the GATE calculator (one-page Microsoft Excel format) and then transferred the calculated results to the GATE-lite form (one-page Microsoft Word format) [46]. We used GATE to illustrate individual study designs and study details as recommended for gauging bias risks [56].

We illustrated the study design using the acronym PECOT, i.e., extracted data on participants, exposure, comparisons, outcomes, and time. To assess study validity, we used the acronym RAMBOMAN, i.e., extracted data on recruitment, allocation, maintenance, blind or objective measurements, and analyses.

We applied the GATE approach to a total of eight non-meta-analyzed cross-sectional studies $[9,22-25$, 53-55] that investigated asthma prevalence or asthma symptoms. The studies included a total of 4,155 children with current asthma/or wheezing, and the total number of participants was 41,770 children (Table 4 and Additional file 4). Unfortunately, in one study [23], the absolute number of participants was not given. The prevalence of asthma/or wheezing in six of these studies 
Table 4 Cross-Sectional Studies Included in the Systematic Review by Selected Study Information

\begin{tabular}{|c|c|c|c|c|c|c|c|c|c|c|}
\hline \multirow[t]{2}{*}{ Author } & \multirow[t]{2}{*}{ Year } & \multirow{2}{*}{$\begin{array}{l}\text { Study } \\
\text { design }\end{array}$} & \multirow{2}{*}{$\begin{array}{l}\text { Age* } \\
\text { (years) }\end{array}$} & \multicolumn{2}{|l|}{ Definition } & \multicolumn{2}{|l|}{ Number } & \multirow[t]{2}{*}{ Main effect size Adj OR } & \multirow[t]{2}{*}{ Adj covariates } & \multirow{2}{*}{$\begin{array}{l}\text { Key conclusions } \\
\text { reported by the } \\
\text { study authors }\end{array}$} \\
\hline & & & & Physical activity & Asthma & Asthma & Total & & & \\
\hline Nystad & 1997 & $\begin{array}{l}\text { Cross- } \\
\text { sectional }\end{array}$ & $\begin{array}{c}7-16 \\
\text { Area I- } \\
\text { III }\end{array}$ & $\begin{array}{l}\text { HBSC (WHO), } \\
\text { two questions } \\
\text { (hrs/wk and } \\
\text { freq/wk) }\end{array}$ & $\begin{array}{l}\text { ISAAC questionnaire } \\
\text { and question on } \\
\text { current asthma from } \\
\text { reference }\end{array}$ & $\begin{array}{l}222 \text { Area I: } \\
123 \text { I: } 69 \text { III: } \\
30\end{array}$ & $\begin{array}{l}\text { 4,021 Area I: } \\
\text { 2,188 II: } \\
\text { 1,045 III: } 788\end{array}$ & $\begin{array}{l}\text { Association (OR [95\%CI]) between } \\
\text { current asthma and PA 1-3 hrs/wk vs } \\
\leq 0.5 \mathrm{hr} / \mathrm{wk}(\mathrm{rfgr}): 1.0(0.6-1.5)\end{array}$ & Age, gender, study area & $\begin{array}{c}\text { Asthmatic children } \\
\text { as physically active } \\
\text { as peers }\end{array}$ \\
\hline Nystad & 2001 & $\begin{array}{l}\text { Cross- } \\
\text { sectional }\end{array}$ & $7-16$ & $\begin{array}{l}\text { HBSC }(\text { WHO), } \\
\text { two questions } \\
\text { (hrs/wk and } \\
\text { freq/wk); only } \\
\text { hrs reported in } \\
\text { article }\end{array}$ & $\begin{array}{l}\text { ISAAC questionnaire } \\
\text { plus question about } \\
\text { current asthma } \\
\text { (from ATS-MRC) }\end{array}$ & $116^{\mathrm{wz}}$ & 2,112 & $\begin{array}{c}\text { Associations (OR [95\%Cl]) wz or } \\
\text { whistling (all children) and PA } \leq 1 \mathrm{hr} / \mathrm{wk} \text { : } \\
1.9 \text { (0.9-3.8)2-3 hrs/wk: } 2.6(1.3-5.2) \geq 4 \\
\text { hrs/wk: } 2.5 \text { (1.2-4.9)vs none (rfgr) "No } \\
\text { clear dose-response relationship, but the } \\
\text { effect was mainly among active vs } \\
\text { inactive children" }\end{array}$ & $\begin{array}{l}\text { Age, atopy (eczema and/or hay } \\
\text { fever), current asthma, gender }\end{array}$ & $\begin{array}{c}\text { Positive } \\
\text { associations } \\
\text { between PA and } \\
\text { WZ }\end{array}$ \\
\hline Lang & 2004 & $\begin{array}{l}\text { Cross- } \\
\text { sectional }\end{array}$ & $6-12$ & $\begin{array}{l}\text { Questionnaire 1) } \\
\text { total mins } \\
\text { active in one (1) } \\
\text { day; } 2 \text { ) number } \\
\text { of days active in } \\
\text { typical wk }\end{array}$ & $\begin{array}{l}\text { Questionnaire. } \\
\text { Medical provider } \\
\text { ever-diagnosed } \\
\text { asthma and some } \\
\text { asthma symptoms in } \\
\text { last } 12 \text { months }\end{array}$ & 137 & 243 & $\begin{array}{c}\text { Association (OR [95\%CI]) between mod/ } \\
\text { severe persistent asthma and PA <30 } \\
\text { mins/day (inactivity) vs all other PA- } \\
\text { groups (ffgr): } 3.00(1.19-7.52)(P<0.05)\end{array}$ & $\begin{array}{l}\text { Gender, health beliefs (e.g., } \\
\text { child can do as much PA as } \\
\text { children similar age without } \\
\text { asthma or child upset with } \\
\text { strenuous activity) }\end{array}$ & $\begin{array}{l}\text { Disease severity } \\
\text { and parental } \\
\text { health beliefs } \\
\text { contributed to } \\
\text { lower activity } \\
\text { levels of children } \\
\text { with asthma }\end{array}$ \\
\hline Jones & 2006 & $\begin{array}{l}\text { Cross- } \\
\text { sectional }\end{array}$ & $\begin{array}{l}\text { 9-12th } \\
\text { grade }\end{array}$ & $\begin{array}{c}\text { PA-levels } \\
\text { (questionnaire) }\end{array}$ & $\begin{array}{c}\text { Questionnaire. } \\
\text { Physician-diagnosed } \\
\text { asthma denoted } \\
\text { lifetime asthma with/ } \\
\text { without current } \\
\text { asthma last } 12 \\
\text { months }\end{array}$ & 1,943 & 13,553 & $\begin{array}{c}\text { Association (OR }[95 \% \mathrm{CI}] \text { ) between } \\
\text { asthma status and sufficient mod PA: } \\
1.1(0.9-1.3)\end{array}$ & Grade, race/ethnicity, gender & $\begin{array}{l}\text { No differences in } \\
\text { participation in vig } \\
\text { or mod PA among } \\
\text { students with and } \\
\text { without current } \\
\text { asthma }\end{array}$ \\
\hline Priftis & 2007 & $\begin{array}{l}\text { Cross- } \\
\text { sectional }\end{array}$ & $10-12$ & $\begin{array}{l}\text { PA questionnaire } \\
\text { (PANACEA) }\end{array}$ & $\begin{array}{l}\text { ISAAC questionnaire. } \\
\text { Asthma symptoms, } \\
\text { e.g., ever asthma or } \\
\text { ever wz }\end{array}$ & $166^{\text {Symptoms }}$ & 700 & $\begin{array}{c}\text { Associations (OR [95\%CI]) for asthma } \\
\text { symptoms in boys; girls not participating } \\
\text { in any PA vs no participation last wk } \\
\text { (Ifgr): } 2.17(1.34-3.54)(P<0.05) ; 1.63 \\
(0.86-3.11)\end{array}$ & $\begin{array}{c}\text { Body weight (per } 5 \mathrm{~kg} \text { ), time of } \\
\text { watching TV or playing video } \\
\text { games per day } \\
\text { (per } 1 \mathrm{hr} \text { ) }\end{array}$ & $\begin{array}{l}\text { PA associated with } \\
\text { reduced odds of } \\
\text { reporting asthma } \\
\text { symptoms }\end{array}$ \\
\hline Corbo & 2008 & $\begin{array}{l}\text { Cross- } \\
\text { sectional }\end{array}$ & $6-7$ & $\begin{array}{l}\text { PA levels in } \\
\text { regular sports (i.e., } \\
\text { formal games or } \\
\text { other aerobic } \\
\text { exercise) } \\
\text { (questionnaire) }\end{array}$ & $\begin{array}{l}\text { ISAAC questionnaire. } \\
\text { Defined current } \\
\text { asthma }\end{array}$ & 1,343 & 20,016 & $\begin{array}{l}\text { Association (OR [95\%Cl]) between } \\
\text { current asthma and low freq of regular } \\
\text { sports ( } 1-2 \text { times per wk) vs none (rfgr): } \\
1.13(0.93-1.38)(P \text { trend }=0.069)\end{array}$ & $\begin{array}{c}\text { Age, BMI, dietary variables, } \\
\text { family asthma or rhinitis, mold, } \\
\text { parental education and } \\
\text { smoking, person filling } \\
\text { questionnaire, regular sports, } \\
\text { season, gender, study center, TV } \\
\text { viewing }\end{array}$ & $\begin{array}{l}\text { Wz or asthma not } \\
\text { associated with } \\
\text { regular sports } \\
\text { activity }\end{array}$ \\
\hline Kosti & 2012 & $\begin{array}{l}\text { Cross- } \\
\text { sectional }\end{array}$ & $10-12$ & $\begin{array}{l}\text { PA questionnaire } \\
\text { (PANACEA) }\end{array}$ & $\begin{array}{l}\text { ISAAC questionnaire. } \\
\text { Asthma symptoms, } \\
\text { e.g., ever asthma or } \\
\text { ever wz }\end{array}$ & 228 & 1,125 & $\begin{array}{l}\text { Association (OR [95\%Cl]) between } \\
\text { leisure-time PA and asthma } \\
\text { symptoms: } 0.90(0.79-1.03)(\mathrm{Ns})\end{array}$ & $\begin{array}{l}\text { Age, BMI, KIDMORE score, } \\
\text { gender, urban/rural }\end{array}$ & $\begin{array}{l}\text { Inverse } \\
\text { relationship } \\
\text { between asthma } \\
\text { symptoms and } \\
\text { leisure PA (rural) }\end{array}$ \\
\hline
\end{tabular}


Table 4 Cross-Sectional Studies Included in the Systematic Review by Selected Study Information (Continued)

\begin{tabular}{|c|c|c|c|c|c|c|c|c|c|c|}
\hline Mitchell & 2013 & $\begin{array}{l}\text { Cross- } \\
\text { sectional }\end{array}$ & $\begin{array}{c}6-7 \\
\text { and } \\
13-14\end{array}$ & $\begin{array}{c}\text { Weekly vig PA } \\
\text { (freq) } \\
\text { (questionnaire) }\end{array}$ & $\begin{array}{l}\text { ISAAC questionnaire, } \\
\text { i.e., ever asthma }\end{array}$ & $\begin{array}{l}\text { Data not } \\
\text { given }\end{array}$ & $\begin{array}{c}76,164(6-7 \\
\text { years)201,370 } \\
(13-14 \text { years) }\end{array}$ & $\begin{array}{c}\text { Associations (OR }[95 \% \mathrm{Cl}] \text { ) between } \\
\text { reported asthma ever and PA once } \\
\text { or twice per wk vs vig PA never or } \\
\text { occasionally each wk (rfgr): } 0.96 \\
(0.89-1.04)(6-7 \text { years) } 1.14 \\
(1.08-1.20)(13-14 \text { years) }\end{array}$ & $\begin{array}{l}\text { BMI, income, language, region, } \\
\text { gender, TV viewing }\end{array}$ & $\begin{array}{l}\text { Vig PA positively } \\
\text { associated with } \\
\text { symptoms of } \\
\text { asthma in } \\
\text { adolescents but } \\
\text { not in children }\end{array}$ \\
\hline
\end{tabular}

Adj Adjusted or adjustment, *Age: Range, ATS-MRC American Thoracic Society and Medical Research Council, BMI Body mass index, Cl Confidence interval, Freq Frequency, Hr/hrs Hour/hours, HBSC Health Behaviour in School-aged Children, ISAAC International Study of Asthma and Allergies in Childhood (ever asthma and wz last 12 months) [2], KIDMORE index Mediterranean Diet Quality Index for children and adolescents (total scores and categories described in article), Min/s Minute/s, Mod Moderate, Ns Non-significant, OR Odds ratio, PA Physical activity, PANACEA The Physical Activity, Nutrition and Allergies in Children Examined in Athens Study, Rfgr Reference group, Vig Vigorous, Vs Versus, Wk Week, Wz Wheezing 
Table 5 Distribution (N, \%) of Children with New-Onset Asthma/or Wheezing and All Children According to PA

\begin{tabular}{|c|c|c|c|c|}
\hline $\begin{array}{l}\text { First author, } \\
\text { publication year }\end{array}$ & PA-exposure levels & New-onset asthma outcome & $\begin{array}{l}\text { New-onset } \\
\text { asthma, N (\%) }\end{array}$ & All children N (\%) \\
\hline Vogelberg et al., 2007 [52] & $\begin{array}{l}\text { Low PA if sport frea } \leq \text { once/wk } \\
\text { High PA (rfgr) if sport freq } \geq \text { two times/wk }\end{array}$ & Wheezing & $\begin{array}{l}\text { Low PA: } 199 \text { (60.5) } \\
\text { High PA: } 130(39.5)\end{array}$ & $\begin{array}{l}\text { Low PA: } 1,470(51.4) \\
\text { High PA: } 1,388(48.6)\end{array}$ \\
\hline Sherriff et al., 2009 [45] & $\begin{array}{l}\text { Low PA if TV viewing } \geq 1 \mathrm{hr} / \text { day } \\
\text { High PA (rfgr) if TV viewing = none or }<1 \mathrm{hr} / \text { day }\end{array}$ & Asthma & $\begin{array}{l}\text { Low PA: } 61 \text { (78.2) } \\
\text { High PA: } 17(21.8)\end{array}$ & $\begin{array}{l}\text { Low PA: 1,100 (68.8) } \\
\text { High PA: } 499(31.2)\end{array}$ \\
\hline Islam et al., 2009 [51] & $\begin{array}{l}\text { Low PA if number of team sports }=\text { none } \\
\text { High PA (rfgr) if number of team sports } \geq 1\end{array}$ & Asthma & $\begin{array}{l}\text { Low PA: } 57(40.1) \\
\text { High PA: } 85 \text { (59.9) }\end{array}$ & $\begin{array}{l}\text { Low PA: } 648 \text { (41.0) } \\
\text { High PA: } 932 \text { (59.0) }\end{array}$ \\
\hline
\end{tabular}

Details regarding data from the meta-analyzed studies

Freq Frequency, Hr/hrs Hour/hours, N Number, PA Physical activity, Rfgr Reference group, Wk Week

$[9,24,25,53-55]$ ranged from $3.8 \%[55]$ to $23.7 \%$ [24] (Table 4). Five cross-sectional studies originated from Europe $[9,24,25,54,55]$, and two were from North America [22, 53]. One study was cross-national [23] and included data for 6-7-year-olds from 17 countries and data for 13-14-year-olds from 35 countries (Additional file 4). A majority of the eligible populations were derived from respective national surveys $[9,24,25,53-55]$ of children and adolescents (Additional file 4).

The GATE assessment showed that all eight studies $[9,22-25,53-55]$ included measures of exposure and outcome and included a comparison group, and all authors reported the results of adjusted analyses; however, for six studies [22-25, 53, 54], we were unable to obtain data on either the exposure or the comparison groups (Additional file 4). The response rates were $>50 \%$ in seven $[9,23-25,53-55]$ of the eight studies, although Mitchell et al. [23] observed a response rate $<50 \%$ for younger children (6-7 years of age) (Additional file 4). Two cross-sectional studies $[22,53]$ analyzed PA as an outcome.

The definitions of PA varied. Nystad [55] and Nystad et al. [9] measured PA outside of school hours (sports or exercise) that caused a child to become sweaty or out of breath. Lang et al. [22] registered the total minutes spent engaging in PA in one day, Jones at al. [53] assessed

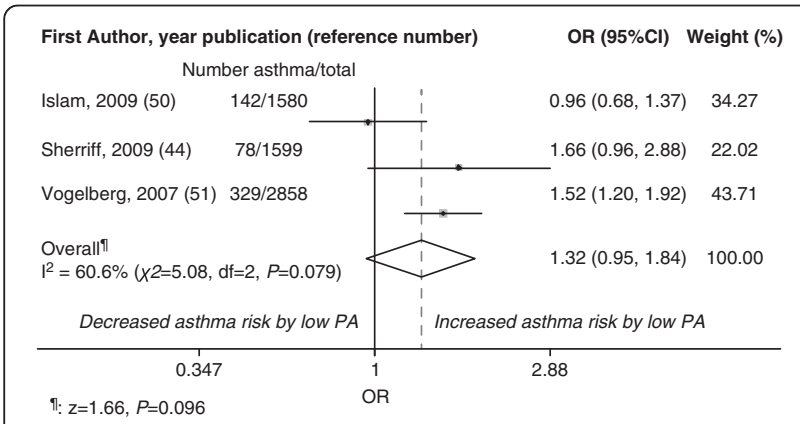

Fig. 2 Random-Effects Model: Study-Specific and Overall Odds Ratios (ORs) with $95 \%$ Confidence Intervals (Cls). Data are derived from the meta-analysis of low physical activity (PA) and new-onset asthma during childhood. High PA: Reference category sufficient moderate PA (e.g., fast walking, slow bicycling), and Priftis et al. [24] examined sports-related PA (e.g., brisk walking, running, swimming). Corbo et al. [25] registered PA as regular sports, i.e., formal games or forms of aerobic exercise. Kosti et al. [54] observed leisure-time PA, i.e., unstructured outdoor PA involving play, walking, or cycling. Mitchell et al. [23] described PA as weekly vigorous activity that was sufficient to cause heavy breathing in the child.

Additionally, the definition of low PA varied, with some studies defining low PA as $\leq 1$ hour per week [9], $<30$ min per day [22], once or twice per week [23], no participation in any PA [24], sports 1-2 times per week [25], sufficient moderate PA [53], leisure-time PA [54], and $1-3 \mathrm{~h}$ per week [55]. In four [9, 24, 25, 55], one [23], and one [54] of the eight [9, 22-25, 53-55] crosssectional studies, the reference groups in the adjusted analyses were "low to no PA", "no vigorous PA", or "no leisure time", respectively (Additional file 4).

In four of the eight studies $[9,22,53,55]$, we were able to extract data for the GATE calculator to estimate occurrences in exposure groups and/or exposure effects. In the studies that investigated distinct low PA $(\leq 1 \mathrm{~h}$ per week [9], 1-3 h per week [55], <30 min per day [22]), the occurrences per 100 persons in the exposure groups (EGO) were 6.2 [9], 4.1 [55], and 14.6 [22]. Two [9, 55] of these studies provided sufficient data to estimate exposure

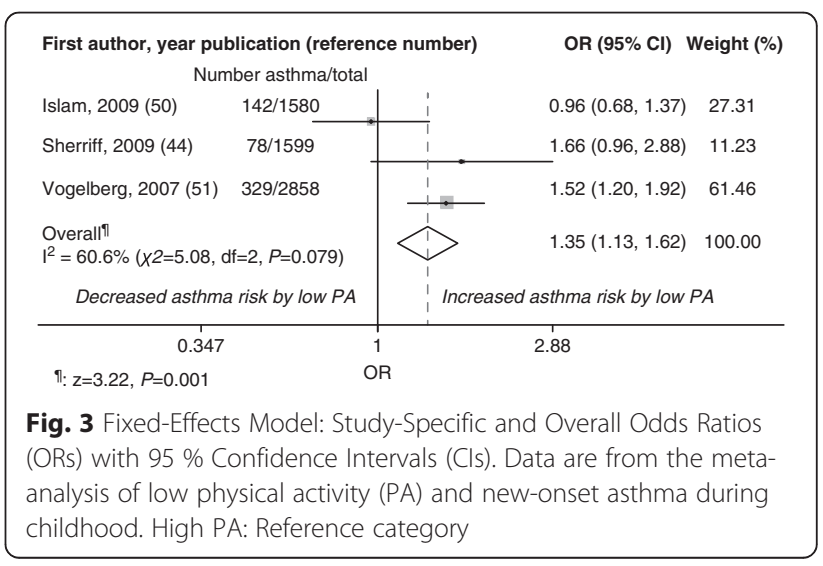




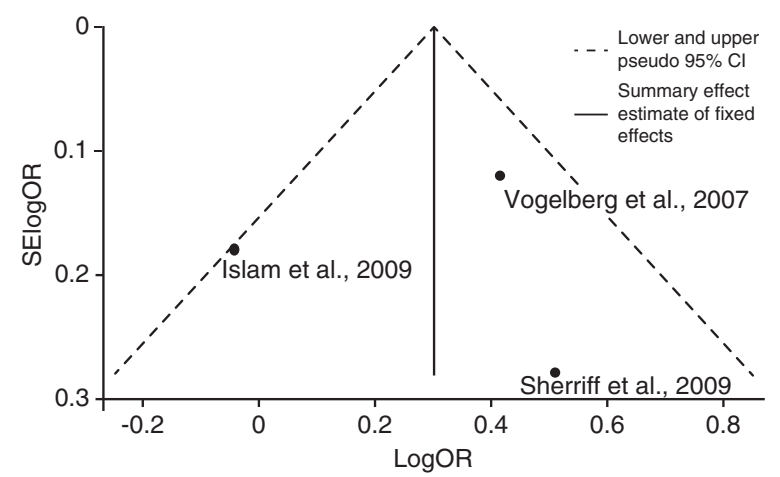

Fig. 4 Funnel Plot with $95 \%$ Pseudo Confidence Intervals (Cls). Data are from the meta-analysis depicting the log-scale odds ratios (logORs) (horizontal axis) for new-onset childhood asthma by low physical activity (PA) using individual study effect size data plotted against the standard errors (SEs) (vertical axis) of the logORs

effects in terms of relative risk (RR) (Additional file 4). In the remaining four studies $[23-25,54]$, we could not derive appropriate data for the calculations.

Although we found each of the eight cross-sectional studies $[9,22-25,53-55]$ applicable to practice (Additional file 4), the GATE analysis illustrated variations across the studies. We concluded that the quality of these studies was high for cross-sectional designs, but the variation among the studies confirmed that individual study analysis (e.g., GATE assessment) as opposed to common estimation across studies (e.g., meta-analysis) was a sound approach that agreed with recommendations $[29,49,57]$.

\section{All studies - measurements PA}

In all but one study, PA was assessed using a questionnaire that asked about sports participation (Tables 3 and 4). One cohort study [45] reported TV viewing (Table 3).

\section{Main effect size and adjustment covariates}

Tables 3 and 4 show that six studies reported positive associations between asthma/or wheezing and low PA [9, 22, 24, 25, 45, 53], and one study showed that asthma/or wheezing was positively associated with high PA [51]. Of the eight cross-sectional studies $[9,22-25,53-55]$, three [22-24] indicated significant positive associations between childhood asthma or asthma symptoms and low PA (of which one [23] reported this association for 13- to 14-year-olds only and one [24] reported this association for boys only).

The adjustment covariates applied in the multivariate analyses varied across the reviewed studies. All authors adjusted for age, gender, weight, and/or smoking measures (Tables 3 and 4), three studies included adjustments for asthma history $[9,25,45]$, and eight studies adjusted for socioeconomic measures [23, 25, 45, 51-55].

\section{Summarizing meta-analysis and GATE review}

In each section, we first reported the descriptive data syntheses and then the analytical data syntheses based on quantitative (meta-analysis) and qualitative (GATE) approach. Meta-analysis was applied to three cohort studies while the GATE assessment was used to assess eight cross-sectional study designs.

Children and adolescents with low PA had increased risk of new-onset asthma, and some showed a higher risk of current asthma/or wheezing, but we found variations among the studies.

\section{Discussion}

The cohort studies showed that the overall risks of newonset asthma/or wheezing increased up to $35 \%$ in children with low PA, and three cross-sectional studies showed significant positive associations with low PA. Of the 11 studies we reviewed, more than $50 \%$ suggested positive associations between childhood asthma and low PA. The critical problem was variation across the reviewed studies. We therefore applied appropriate epidemiological methods when performing meta-analysis of similar studies and when graphically assessing those that were dissimilar.

This systematic review followed established guidelines [29]. The review included $>500$ cases of new-onset (incident) asthma/or wheezing and approximately 4,000 current (prevalent) asthma cases. Although the number of studies was moderate, the inclusion of a variety of study designs may be advantageous. Previous investigations have produced contradictory results for the association under study, and the cross-sectional study design has limitations with respect to ruling out the directions of associations; therefore, we sought to identify studies with a longitudinal design. The longitudinal design of cohort studies overcomes the limitations of the cross-sectional design because measures of cause and effect are separated in time. Reverse causation (i.e., the notion that asthma causes low PA) was accounted for by the cohort studies [45, 51, 52]. For example, one study [45] included only asymptomatic children. Hence, we were able to derive some assessment of the directions of the associations. Other authors $[58,59]$ have proposed hypotheses fairly similar to ours; this review could confirm significant positive associations described by three [22-24] cross-sectional and two longitudinal $[45,52]$ studies.

The intensity of leisure-time activity studied by Vogelberg et al. [52] was similar to that of organized team sports studied by Islam et al. [51]. Although leisure-time activity differs from organized sports [37], they both fall along a spectrum of aerobic activities. The leisure activities included, e.g., running, bicycling, and swimming [52,60], and 
the team sports encompassed a range of intensity from low to high $[51,61]$. Thus, we could not identify systematic deviations in the PA definitions of these two studies $[51,52]$.

Generally, the quality of the reviewed studies was high. Although GATE does not provide one single quantitative assessment score [62], the observational studies appeared to reflect good standards for internal validity. We excluded ecological studies in an effort to retrieve studies with a rigorous design [63]. Although the clinical application of reviews is often overlooked [47], our results appear to align with those of others who have acknowledged the clinical importance of observational studies [64].

Recent systematic reviews have investigated the prevalence of wheezing in children [65] or PA in adolescents [66], but few have reviewed both. The asthma diagnosis was critical for our results. Asthma is a heterogeneous clinical syndrome [67], and because the diagnosis of asthma in children lacks a gold standard, it is ideally verified by uniform guidelines [68]. The asthma definitions in the current review were relatively uniform. Seven of the eleven studies used physician-confirmed asthma diagnoses, and our review populations were homogeneous (Europe and North America). Earlier reviews [65] that had to rely on less rigorous asthma symptom reports lack these characteristics.

All reviewed studies performed PA quantification. The cross-national survey, for example, used the ISAAC questionnaire [23] and showed a significant association between asthma and low PA in adolescents but not in children. Data on activity in young children are often difficult for parents to report, and in fact, some of the cross-sectional studies included 6-year-olds. The younger children in these studies received parental assistance with the questionnaire, and thus we cannot rule out information bias. Recent evidence has certainly suggested that parents and peers influence $\mathrm{PA}$ in both healthy [69-71] and asthmatic [72] children. Although we recognize that accelerometry still requires technical improvements for optimal use in the youngest children [71, 73, 74], the reported findings appear to align with earlier objective measurements that employed accelerometry [59].

The strength of the effect sizes varied, and the smaller studies [22, 24] yielded larger effect sizes, as expected. Moreover, low PA varied; for example, Lang et al. [22] analyzed daily PA durations as low as 30 min. Analogously, Nystad et al. [9] quantified "very low" PA ( $<1 \mathrm{~h} /$ week). Lang et al. [22] measured PA during the school day, whereas Nystad et al. [9] studied PA outside of school hours. This diurnal variation in PA could be of importance to the results because energy expended during seated school-day activities varies from that expended during leisure PA [75]. The reporting of PA also varied and included duration and frequency. Although protective associations between PA and current asthma were non-significant, Nystad [55] suggested that these associations could be a factor when PA frequency is analyzed. Therefore, although PA frequency and duration show correlations in children [76], it may be relevant to report both.

Our review may have certain limitations. Formal meta-analysis of the cross-sectional studies was not reasonable given that the overestimation of effects is well documented [57]. Although GATE is only one of a number of existing quality appraisal tools [62], we acknowledge that it provided some systematization to our assessments.

Limitations of the calculations were also made evident when individual patient data were not provided in the articles. We lacked some data on the exposed and nonexposed groups described in the cross-sectional studies. Although statistical methodology exists for imputing data [47], no such technique was used in the current analyses. Our meta-analysis was a two-stage process [48]. The first results produced were the summary statistics of each individual study that was included. These results agreed with the conclusions of each study. Because the meta-analysis inclusion criteria were met, we then combined the statistics. We have discussed the variation in the exposures (PA) and outcomes (asthma/or wheezing) of the meta-analyzed studies $[45,51,52]$, but the basic cohort methodologies also appeared to be rather similar.

The meta-analysis revealed increased risks of newonset asthma among children who reported low PA. The funnel plot showed that these three studies lay within the confidence intervals; this illustration may favor limited heterogeneity. Although we must expect some inter-study variability, the random-effects model could have assigned disproportionate influence to the studies with the smallest sample sizes. We cannot draw firm conclusions from the limited number of cohort studies available, but the parallel results for the fixed and random estimations may indicate only modest heterogeneity. We generated I-squared values of approximately $60 \%$ (with non-significant chi-squared tests), and based on current guidelines [77], these findings may support our assumptions regarding heterogeneity. In the random- and fixed-effects models, this result implied that $60 \%$ of the between-study heterogeneity could be explained by true study variation $[47,78]$.

Body composition was not reviewed in this study. Although obesity is related to childhood asthma [79, 80], the effects of asthma and weight on lung function are highly variable [81].

Future studies should involve the participation of clinical professionals. Clinicians (e.g., pediatricians or epidemiologists) may find our results useful when inquiring 
about the PA of their young patients who present with respiratory symptoms or asthma.

\section{Conclusions}

Of the 1,571 titles reviewed, we analyzed 11 original articles. Overall, we observed indications that children who were physically inactive may have a higher risk of asthma/or wheezing compared with active children. This review also revealed a critical need for future longitudinal assessments of low PA, its mechanisms, and its implications for incident asthma in children.

\section{Additional files}

Additional file 1: PRISMA Items Used in Reporting in the Current Systematic Literature Review. Additional file 1 presents the PRISMA checklist items that were examined, with the draft article page numbers. (DOCX 29 kb)

Additional file 2: Reporting Items of the Meta-analysis Of Observational Studies in Epidemiology (MOOSE) Group. (PDF 532 kb)

Additional file 3: A. Articles Excluded $(n=54)$ from the Systematic Review Grouped by Exclusion Rationale. B. Articles Excluded $(n=7)$ from the Systematic Review after Review of the Reference Lists. (DOCX 25 kb)

Additional file 4: Data from Eight Non-Meta-Analyzed Studies Extracted to the GATE Calculator and the GATE-Lite Appraisal Forms. (PDF $8281 \mathrm{~kb}$ )

\section{Abbreviations}

BHR: bronchial hyperresponsiveness/or bronchial hyperresponsibility BMI: body mass index; Cl: confidence interval; ElA: exercise-induced asthma; GATE: Graphic Appraisal Tool for Epidemiology; HR: hazard ratio; ISAAC: International Study of Asthma and Allergies in Childhood; MeSH: medical subject heading; MET: metabolic equivalent; OR: odds ratio; PA: physical activity; RCT: randomised controlled trial; SE: standard error; SES: socio-economic status.

\section{Competing interests}

The authors declare that they have no competing interests.

\section{Authors' contributions}

$\mathrm{LL}$ developed the protocol, performed the literature searches, extracted the data for quantitative and qualitative syntheses, and conducted the data analyses. PEP was available for adjudication. KGN, PEP, and LL developed the strategy for GATE application and interpreted the results with assistance from RTJ. All authors (PEP, TAEPM, KGN, and LL) participated in the PROSPERO registration (University of York, UK) and are joint guarantors of this review. All authors also contributed to assessing the comprehensive results. LL drafted the initial manuscript, KGN and PEP revised the drafts critically, and all authors read and accepted the final manuscript.

\section{Acknowledgements}

The review commenced during LL's initial research program at the University of Bristol (UoB), Bristol, United Kingdom. Therefore, the authors wish to express thanks to Medical Subject Librarian CF Borwick (CFB), UoB, for assistance in refining the database search strategies. We also thank Prof RT Jackson (RTJ) of the University of Auckland (Auckland, New Zealand) for reviewing our data, providing helpful guidance (which included providing savable files for GATE), and offering suggestions regarding our application of the GATE tool.

\section{Funding}

$\mathrm{LL}$ received funding from one PhD Fellowship and two research awards (respective award dates: 13 May 2009, 9 Dec 2010, and 14 June 2011) from The Jubilee Foundation* and from four research grants of The Research Fund* dated 14 May 2008, 13 May 2009, 14 June 2011, and 10 Dec 2013.
The funders had no role in the study design, data collection and analysis, decision to publish, or preparation of the manuscript.

*Danish Physiotherapy Organization

\section{Author details}

'Department of Odontology, University of Copenhagen, Copenhagen 1014 Denmark. ${ }^{2}$ Department of Pediatrics and Adolescent Medicine, Copenhagen University Hospital, Rigshospitalet, Copenhagen 2100, Denmark. ${ }^{3}$ Department of Medicine, Division of Allergy and Clinical Immunology, University of Virginia, Charlottesville 22908, VA, USA.

Received: 18 September 2015 Accepted: 8 March 2016

Published online: 18 April 2016

\section{References}

1. Papadopoulos NG, Arakawa H, Carlsen KH, Custovic A, Gern J, Lemanske R, et al. International consensus on (ICON) pediatric asthma. Allergy. 2012;67: 976-97.

2. Asher M, Keil U, Anderson H, Beasley R, Crane J, Martinez F, et al. International Study of Asthma and Allergies in Childhood (ISAAC): rationale and methods. Eur Respir J. 1995;8:483-91.

3. Burney PGJ, Chinn S, Rona RJ. Has the prevalence of asthma increased in children? Evidence from the National Study of Health and Growth 1973-86. BMJ. 1990:300:1306-10.

4. Ross Anderson H, Gupta R, Strachan DP, Limb ES. 50 years of asthma: UK trends from 1955 to 2004. Thorax. 2007:62:85-90.

5. Hill VL, Wood PR. Asthma epidemiology, pathophysiology, and initial evaluation. Pediatr Rev. 2009:30:331-6.

6. Asher M, Montefort S, Bjorksten B, Lai C, Strachan D, Weiland S, et al. Worldwide time trends in the prevalence of symptoms of asthma, allergic rhinoconjunctivitis, and eczema in childhood: ISAAC Phases One and Three repeat multicountry cross-sectional surveys. Lancet. 2006;368:733-43.

7. Platts-Mills TAE. Asthma severity and prevalence: an ongoing interaction between exposure, hygiene, and lifestyle. PLoS Med. 2005;2:e34.

8. Subbarao P, Mandhane PJ, Sears MR. Asthma: epidemiology, etiology and risk factors. CMAJ. 2009;181:E181-90.

9. Nystad W, Nafstad P, Harris J. Physical activity affects the prevalence of reported wheeze. Eur J Epidemiol. 2001;17:209-12

10. Shaaban R, Leynaert B, Soussan D, Antó JM, Chinn S, de Marco R, et al. Physical activity and bronchial hyperresponsiveness: European Community Respiratory Health Survey II. Thorax. 2007;62:403-10.

11. World Health Organization. Children's environmental health: Children, mobility and environmental health. World Health Organization. http://www.who.int/ ceh/risks/en/. Accessed 30 Mar 2016

12. Cypcar D, Lemanske Jr RF. Asthma and exercise. Clin Chest Med. 1994;15: 351-68.

13. Matsumoto I, Araki H, Tsuda K, Odajima H, Nishima S, Higaki Y, et al. Effects of swimming training on aerobic capacity and exercise induced bronchoconstriction in children with bronchial asthma. Thorax. 1999:54:196-201.

14. Welsh L, Kemp JG, Roberts RGD. Effects of physical conditioning on children and adolescents with asthma. Sports Med. 2005;35:127-41.

15. Schwindt CD, Zaldivar F, Wilson L, Leu S-Y, Wang-Rodriguez J, Mills PJ, et al. Do circulating leucocytes and lymphocyte subtypes increase in response to brief exercise in children with and without asthma? Br J Sports Med. 2007:41:34-40.

16. Rosa J. Exercise leukocyte profiles in healthy, type 1 diabetic, overweight, and asthmatic children. Pediatr Exerc Sci. 2009;21:19-33.

17. Lucas SR, Platts-Mills TAE. Physical activity and exercise in asthma: relevance to etiology and treatment. J Allergy Clin Immunol. 2005;115: 928-34

18. Cooper DM, Radom-Aizik S, Schwindt C, Zaldivar Jr F. Dangerous exercise: lessons learned from dysregulated inflammatory responses to physical activity. J Appl Physiol. 2007:103:700-9.

19. Platts-Mills TAE, Woodfolk JA, Chapman MD, Heymann PW. Changing concepts of allergic disease: The attempt to keep up with real changes in lifestyles. J Allergy Clin Immunol. 1996;98:S297-306.

20. Eijkemans M, Mommers M, de Vries SI, van Buuren S, Stafleu A, Bakker I, et al. Asthmatic symptoms, physical activity, and overweight in young children: a cohort study. Pediatrics. 2008;121:e666-72.

21. Siersted HC, Boldsen J, Hansen HS, Mostgaard G, Hyldebrandt N, Rees PJ, et al. Population based study of risk factors for underdiagnosis of asthma in adolescence: Odense schoolchild study. BMJ. 1998;316:651-7. 
22. Lang DM, Butz AM, Duggan AK, Serwint JR. Physical activity in urban school-aged children with asthma. Pediatrics. 2004;113:e341-6.

23. Mitchell E, Beasley R, Björkstén B, Crane J, García-Marcos L, Keil U. The association between $\mathrm{BMl}$, vigorous physical activity and television viewing and the risk of symptoms of asthma, rhinoconjunctivitis and eczema in children and adolescents: ISAAC Phase Three. Clin Exp Allergy. 2013;43:73-84.

24. Priftis K, Panagiotakos D, Antonogeorgos G, Papadopoulos M, Charisi M, Lagona $\mathrm{E}$, et al. Factors associated with asthma symptoms in schoolchildren from Greece: The Physical Activity, Nutrition and Allergies in Children Examined in Athens (PANACEA) study. J Asthma. 2007:44:521-7.

25. Corbo G, Forastiere F, De Sario M, Brunetti L, Bonci E, Bugiani M, et al. Wheeze and asthma in children: associations with body mass index, sports, television viewing, and diet. Epidemiology. 2008;19:747-55.

26. Ownby D, Peterson E, Nelson D, Joseph C, Williams L, Johnson C. The relationship of physical activity and percentage of body fat to the risk of asthma in 8- to 10-year-old children. J Asthma. 2007:44:885-9.

27. Eijkemans $\mathrm{M}$, Mommers M, Draaisma JM, Thijs C, Prins MH. Physical activity and asthma: a systematic review and meta-analysis. PLOS ONE. 2012;7:e50775.

28. Jackson R, Ameratunga S, Broad J, Connor J, Lethaby A, Robb G, et al. The GATE frame: critical appraisal with pictures. Evid Based Med. 2006;11:35-8.

29. Centre for Reviews and Dissemination. Systematic reviews: CRD's guidance for undertaking reviews in health care. York: University of York; 2009.

30. Moher D, Liberati A, Tetzlaff J, Altman DG, The Prisma Group. Preferred reporting items for systematic reviews and meta-analyses: the PRISMA statement. PLOS Med. 2009;6:e1000097.

31. Stroup DF, Berlin JA, Morton SC, Olkin I, Williamson GD, Rennie D, et al. Meta-analysis of observational studies in epidemiology: a proposal for reporting. JAMA. 2000;283:2008-12.

32. Liberati A, Altman DG, Tetzlaff J, Mulrow C, Gøtzsche PC, loannidis JPA, et al. The PRISMA statement for reporting systematic reviews and meta-analyses of studies that evaluate health care interventions: explanation and elaboration. J Clin Epidemiol. 2009;62:e1-e34.

33. Pearce N. Classification of epidemiological study designs. Int J Epidemiol. 2012:41:393-7.

34. Duijts L. Fetal and infant origins of asthma. Eur J Epidemiol. 2012;27:5-14.

35. Lee DA, Winslow NR, Speight AN, Hey EN. Prevalence and spectrum of asthma in childhood. BMJ. 1983;286:1256-8.

36. Sallis JF, Saelens BE. Assessment of physical activity by self-report: status, limitations, and future directions. Res Q Exercise Sport. 2000;71:S1-S14.

37. Howley E. Type of activity: resistance, aerobic and leisure versus occupational physical activity. Med Sci Sports Exerc. 2001;33:S364-S9.

38. Haskell WL. What to look for in assessing responsiveness to exercise in a health context. Med Sci Sports Exerc. 2001;33:S454-S8.

39. Hickmann M, Roberts C, de Matos M. Exercise and leisure-time activities. In: Currie C, Hurrelmann K, Settertobulte W, Smith R, Todd J, editors. Health and health behaviour among young people. Health behaviour in schoolaged children: a WHO Cross-National Study (HBSC) International Report. Copenhagen: World Health Organization; 2000. p. 73-82.

40. Owen N, Healy GN, Matthews CE, Dunstan DW. Too much sitting: the population health science of sedentary behavior. Exerc Sports Sci Rev. 2010;38:105-13.

41. Pettee Gabriel KK, Morrow Jr JR, Woolsey AL. Framework for physical activity as a complex and multidimensional behavior. J Phys Act Health. 2012;9 Suppl 1:S11-S8.

42. Romieu I, Mannino DM, Redd SC, McGeehin MA. Dietary intake, physical activity, body mass index, and childhood asthma in the third national health and nutrition survey (NHANES III). Pediatr Pulmonol. 2004;38:31-42.

43. Moher D, Pham B, Klassen TP, Schulz KF, Berlin JA, Jadad AR, et al. What contributions do languages other than English make on the results of meta-analyses? J Clin Epidemiol. 2000;53:964-72.

44. Higgins JPT, Deeks JJ. Table 7.3.a. Checklist for items to consider in data collection or data extraction. In: Higgins J, Green S, editors. Cochrane handbook for systematic reviews of interventions, Version 5.1.0 [updated March 2011]. Baltimore: The Cochrane Collaboration; 2011. http://handbook. cochrane.org/. Accessed 30 Mar 2016.

45. Sherriff A, Maitra A, Ness AR, Mattocks C, Riddoch C, Reilly JJ, et al. Association of duration of television viewing in early childhood with the subsequent development of asthma. Thorax. 2009;64:321-5.

46. University of Auckland. Evidence-based practice and critical appraisal. Auckland, NZ: University of Auckland. https://www.fmhs.auckland.ac.nz/en/ soph/about/our-departments/epidemiology-and-biostatistics/research/epiq/ 2015-evidence-based-practice-and-cats.html. Accessed 30 Mar 2016.
47. Riley RD, Higgins JPT, Deeks JJ. Interpretation of random effects metaanalyses. BMJ. 2011;342:d549.

48. Deeks JJ, Altman DG, Bradburn MJ. Statistical methods for examining heterogeneity and combining results from several studies in meta-analysis. In: Egger M, Davey Smith G, Altman DG, editors. Systematic reviews in health care: meta-analysis in context. 2nd ed. London: BMJ; 2008. p. 285-312.

49. Liberati A, Altman DG, Tetzlaff J, Mulrow C, Gotzsche PC, loannidis JP, et al. The PRISMA statement for reporting systematic reviews and meta-analyses of studies that evaluate health care interventions: explanation and elaboration. PLOS Med. 2009;6:e1000100.

50. StataCorp. http://www.stata.com/. Accessed 30 Mar 2016.

51. Islam T, Berhane K, McConnell R, Gauderman WJ, Avol E, Peters JM, et al. Glutathione-S-transferase (GST) P1, GSTM1, exercise, ozone and asthma incidence in school children. Thorax. 2009;64:197-202.

52. Vogelberg $C$, Hirsch $T$, Radon $K$, Dressel H, Windstetter $D$, Weinmayr $G$, et al. Leisure time activity and new onset of wheezing during adolescence. Eur Respir J. 2007;30:672-6.

53. Jones S, Merkle S, Fulton J, Wheeler L, Mannino D. Relationship between asthma, overweight, and physical activity among U.S. high school students. J Commun Health. 2006:31:469-78.

54. Kosti R, Priftis K, Anthracopoulos M, Papadimitriou A, Grigoropoulou D, Lentzas $Y$, et al. The association between leisure-time physical activities and asthma symptoms among 10- to 12-year-old children: the effect of living environment in the PANACEA study. J Asthma. 2012:49:342-8.

55. Nystad W. The physical activity level in children with asthma based on a survey among 7-16-year-old school children. Scand J Med Sci Sports. 1997;7:331-5.

56. Wood L, Egger M, Gluud LL, Schulz KF, Jüni P, Altman DG, et al. Empirical evidence of bias in treatment effect estimates in controlled trials with different interventions and outcomes: meta-epidemiological study. BMJ. 2008;336:601-5.

57. Egger M, Davey Smith G, Altman DG. Systematic reviews in health care: meta-analysis in context. London: BMJ Books; 2001.

58. Firrincieli V, Keller A, Ehrensberger R, Platts-Mills J, Shufflebarger C, Geldmaker B, et al. Decreased physical activity among headstart children with a history of wheezing: use of an accelerometer to measure activity. Pediatr Pulm. 2005;40:57-63.

59. Rundle A, Goldstein IF, Mellins RB, Ashby-Thompson M, Hoepner L, Jacobson JS. Physical activity and asthma symptoms among New York City head start children. J Asthma. 2009;46:803-9.

60. Radon K, Dressel H, Hümmer S, Riu E, Nowak D, Weinmayr G, et al. Berufliche allergierisiken-die SOLAR-kohortenstudie. University of Munich. BAuA - Forschungsberichte 2005/Forschungsberichte/Schriftenreihe der BAuA/Publikationen/Bundesanstalt für Arbeitsschutz und Arbeitsmedizin Berufliche Allergierisiken. Accessed 21 Aug 2015.

61. McConnell R, Berhane K, Gilliland F, London SJ, Islam T, Gauderman WJ, et al. Asthma in exercising children exposed to ozone: a cohort study. Lancet. 2002;359:386-91.

62. Voss PH, Rehfuess EA. Quality appraisal in systematic reviews of public health interventions: an empirical study on the impact of choice of tool on meta-analysis. J Epidemiol Commun H. 2013;67:98-104.

63. Pearce N. Epidemiology in a changing world: variation, causation and ubiquitous risk factors. Int J Epidemiol. 2011;40:503-12.

64. Black N. Why we need observational studies to evaluate the effectiveness of health care. BMJ. 1996;312:1215-8.

65. Patel $S$, Jarvelin M-R, Little M. Systematic review of worldwide variations of the prevalence of wheezing symptoms in children. Environ Health. 2008;7:57.

66. Dumith SC, Gigante DP, Domingues MR, Kohl 3rd HW. Physical activity change during adolescence: a systematic review and a pooled analysis. Int J Epidemiol. 2011;40:685-98

67. Eder W, Ege MJ, von Mutius E. The asthma epidemic. New Engl J Med. 2006;355:2226-35

68. The British Thoracic Society and Scottish Intercollegiate Guidelines Network British guideline on the management of asthma. Quick reference guide, guideline no. 141. London: British Thoracic Society, Scottish Intercollegiate Guidelines Network; 2014. http://www.sign.ac.uk/guidelines/fulltext/141/ index.html. Accessed 30 Mar 2016.

69. Alderman BL, Benham-Deal TB, Jenkins JM. Change in parental influence on children's physical activity over time. J Phys Act Health. 2010;7:60-7.

70. Jago R, Page AS, Cooper AR. Friends and physical activity during the transition from primary to secondary school. Med Sci Sports Exerc. 2012;44:111-7. 
71. Warren JM, Ekelund U, Besson H, Mezzani A, Geladas N, Vanhees L. Assessment of physical activity - a review of methodologies with reference to epidemiological research: a report of the exercise physiology section of the European Association of Cardiovascular Prevention and Rehabilitation. Eur J Cardiov Prev R. 2010;17:127-39.

72. Dantas F, Correia M, Silva A, Peixoto D, Sarinho E, Rizzo J. Mothers impose physical activity restrictions on their asthmatic children and adolescents: an analytical cross-sectional study. BMC Public Health. 2014;14:287.

73. Cliff DP, Reilly JJ, Okely AD. Methodological considerations in using accelerometers to assess habitual physical activity in children aged 0-5 years. J Sci Med Sport. 2009;12:557-67.

74. Bornstein DB, Beets MW, Byun W, Mclver K. Accelerometer-derived physical activity levels of preschoolers: A meta-analysis. J Sci Med Sport. 2011;14:504-11.

75. Ainsworth BE, Haskell WL, Whitt MC, Irwin ML, Swartz AM, Strath SJ, et al. Compendium of physical activities: an update of activity codes and MET intensities. Med Sci Sports Exerc. 2000;32:S498-S516.

76. Booth ML, Okely AD, Chey T, Bauman A. The reliability and validity of the physical activity questions in the WHO health behaviour in schoolchildren (HBSC) survey: a population study. Br J Sports Med. 2001;35:263-7.

77. Higgins JPT, Deeks JJ. 9.5.4 Incorporating heterogeneity into random-effects models. In: Higgins J, Green S, editors. Cochrane handbook for systematic reviews of interventions, Version 5.1.0 [updated March 2011]. Baltimore: The Cochrane Collaboration; 2011. http://handbook.cochrane.org/. Accessed 30 Mar 2016.

78. Higgins JPT, Thompson SG, Deeks JJ, Altman DG. Measuring inconsistency in meta-analyses. BMJ. 2003;327:557-60

79. Quinto KB, Zuraw BL, Poon KY, Chen W, Schatz M, Christiansen SC. The association of obesity and asthma severity and control in children. J Allergy Clin Immunol. 2011;128:964-9.

80. Saint-Pierre P, Bourdin A, Chanez P, Daures JP, Godard P. Are overweight asthmatics more difficult to control? Allergy. 2006;61:79-84.

81. Nicolacakis K, Skowronski ME, Coreno AJ, West E, Nader NZ, Smith RL, et al. Observations on the physiological interactions between obesity and asthma. J Appl Physiol. 2008;105:1533-41.

\section{Submit your next manuscript to BioMed Central and we will help you at every step:}

- We accept pre-submission inquiries

- Our selector tool helps you to find the most relevant journal

- We provide round the clock customer support

- Convenient online submission

- Thorough peer review

- Inclusion in PubMed and all major indexing services

- Maximum visibility for your research

Submit your manuscript at www.biomedcentral.com/submit 